\title{
Irregularly branched microvessels as visualized by magnifying endoscopy: a reliable marker for predicting deep submucosal invasion of superficial esophageal squamous cell carcinoma
}

\section{(c) (1) $(:)$}

\section{Authors}

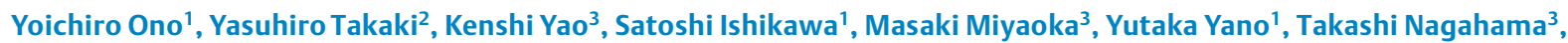
Takashi Hisabe ${ }^{1}$, Fumihito Hirai ${ }^{4}$, Toshiharu Ueki ${ }^{1}$, Atsuko Ota ${ }^{5}$, Hiroshi Tanabe ${ }^{5}$, Seiji Haraoka ${ }^{5}$, Akinori Iwashita ${ }^{5}$, Koji Mikami ${ }^{6}$, Takafumi Maekawa ${ }^{6}$

\section{Institutions}

1 Department of Gastroenterology, Fukuoka University Chikushi Hospital, Fukuoka, Japan

2 Department of Gastroenterology, Ashiya Central Hospital, Kitakyusyu, Japan

3 Department of Endoscopy, Fukuoka University Chikushi Hospital, Fukuoka, Japan

4 Inflammatory Bowel Disease Center, Fukuoka University Chikushi Hospital, Fukuoka, Japan

5 Department of Pathology, Fukuoka University Chikushi Hospital, Fukuoka, Japan

6 Department of Surgery, Fukuoka University Chikushi Hospital, Fukuoka, Japan

submitted 21.12.2018

accepted after revision 22.7.2019

Bibliography

DOI https://doi.org/10.1055/a-1072-4676 |

Endoscopy International Open 2020; 08: E234-E240

(c) Georg Thieme Verlag KG Stuttgart · New York eISSN 2196-9736

Corresponding author Yoichiro Ono, Fukuoka University Chikushi Hospital Gastroenterology, Chikushino Fukuoka 818-8502, Japan Fax: +81929280856

yono6110@gmail.com

\section{ABSTRACT}

Background and study aims Magnifying endoscopy with narrow-band imaging (M-NBI) is reported to be useful in diagnosing invasion depth of superficial esophageal squamous cell carcinoma (SCC), but accurate diagnosis of deep submucosal invasion (SM2) has remained difficult. However, we discovered that irregularly branched microvessels observed with $\mathrm{M}-\mathrm{NBI}$ are detected in SM2 cancers with high prevalence. Thus, this retrospective study aimed to investigate the diagnostic performance of irregularly branched microvessels as visualized by $\mathrm{M}-\mathrm{NBI}$ for predicting SM2 cancers.

Patients and methods Patients with superficial esophageal SCC lesions that were endoscopically or surgically resected at our hospital between September 2005 and December 2014 were included. Endoscopic findings by M-NBI of these lesions were presented to an experienced endoscopist who was unaware of the histopathological diagnosis and who then judged whether irregularly branched microvessels were present. Using the invasion depth according to postoperative histopathological diagnosis as the gold standard, we determined the diagnostic performance of the presence of irregularly branched microvessels as an indicator for SM2 cancers.

Results A total of 302 superficial esophageal SCC lesions (228 patients) were included in the analysis. When irregularly branched microvessels were used as an indicator of SM2 cancers, the diagnostic accuracy was $94.0 \%$ (95\% confidence interval $[\mathrm{Cl}]$ : 91.1-96.1\%), sensitivity was $79.4 \%$ (95\% Cl: 66.6-88.4\%), specificity was $95.9 \%$ (95\% Cl: 94.3-97.0\%), positive predictive value was $71.1 \%$ (95\% Cl: $59.6-79.1 \%$ ), and negative predictive value was $97.3 \%$ (95\% Cl: 95.7-98.5\%).

Conclusions Irregularly branched microvessels may be a reliable $\mathrm{M}-\mathrm{NBI}$ indicator for the diagnosis of cancers with deep submucosal invasion. 


\section{Introduction}

The rate of lymph node metastasis from superficial esophageal squamous cell carcinoma (SCC) has a close relationship to depth of invasion by the cancer [1-7]. According to Japanese guidelines for diagnosis and treatment of esophageal cancer [8], indications for treatment are as follows: an absolute indication for endoscopic resection (ER): EP/LPM cancers; a relative indication for ER: MM/SM1 cancers; and surgical resection/chemoradiotherapy: SM2 cancers. It was reported that the complication rate of surgery for esophageal SCC is $41.9 \%$, with morbidity and mortality rates of $13.3 \%$ and $1.2 \%$ to $3.4 \%$, respectively [9]. Specifically, surgery has a high risk of morbidity and mortality. Therefore, preoperative diagnosis especially focusing on the endoscopic diagnosis of the depth of invasion by the cancer is pertinent to determine the therapeutic strategy.

Furthermore, differential diagnosis between EP-SM1 and SM2 cancers is particularly important to determine whether less invasive ER or invasive surgical treatment should be performed. Magnifying endoscopy with image-enhanced technique is reported to be useful for diagnosis of depth of invasion of superficial esophageal SCC $[10,11]$. According to the magnifying endoscopic classification of the Japan Esophageal Society (JES) [12], the B3 vessels, which are highly dilated abnormal vessels with a caliber often larger than $60 \mu \mathrm{m}$ ( $\triangleright$ Fig. 1 ), are described to be characteristic of SM2 cancers. The specificity of the B3 vessels as a marker for SM2 cancer is high. However, sensitivity was extremely low, which remains an issue [13-15].

In a previous study, Arima et al. reported that the vessels, which are visualized by magnifying endoscopy in cancers with muscularis mucosae or deeper invasion, were dilated and branched out from their origin and formed multiple layers [11]. Similarly, we noticed that irregularly branched microvessels are frequently identified by magnifying endoscopy with narrowband imaging (M-NBI) in invasive SCC. Hence, this study aimed to investigate the diagnostic performance of M-NBI when we employ irregularly branched microvessels as a marker for predicting deep submucosal invasion in superficial esophageal SCC.

\section{Patients and methods}

\section{Patients}

We included consecutive patients with superficial esophageal SCC who received treatments in our hospital between September 2005 and December 2014. Inclusion criteria were as follows: (1) patients who were diagnosed as having superficial esophageal SCC, (2) those who underwent preoperative endoscopic examination using M-NBI; and (3) those whose resected specimen was fully assessed by histopathological investigation. Patients were excluded if they fulfilled the following criteria: (1) patients with a remnant or recurrent lesion after ER; (2) patients with a previously chemotherapy; (3) those with a lesion arising from the irradiated esophageal squamous epithelium; (4) those with a lesion in the remaining esophagus after subtotal esophagectomy; and (5) those with a lesion described as undiagnosable due to poor observational conditions.

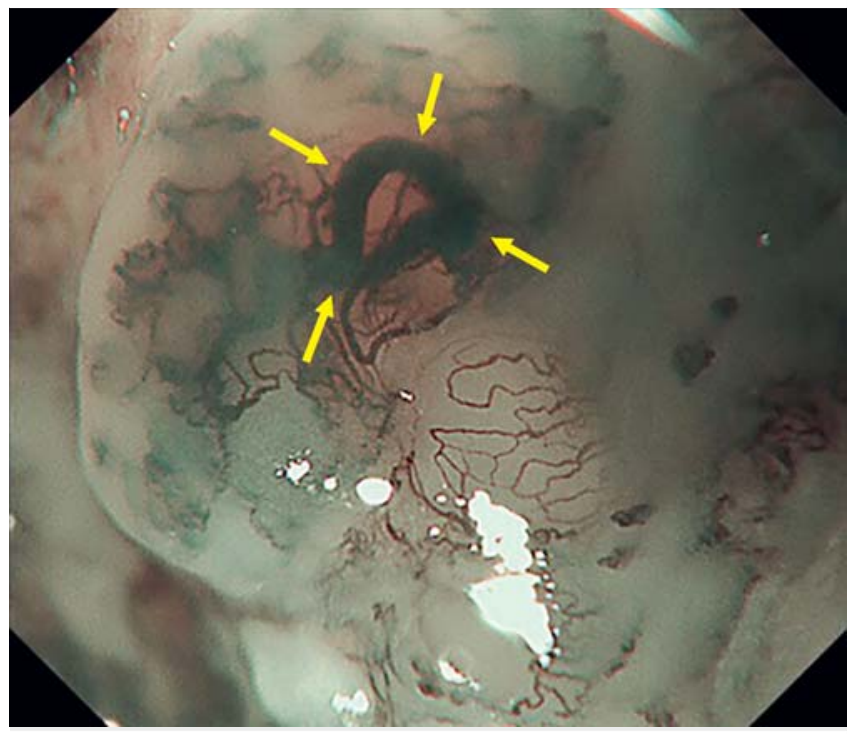

D Fig. 1 B3 vessels of highly dilated abnormal vessels (yellow arrows) with a caliber often larger than $60 \mu \mathrm{m}$.

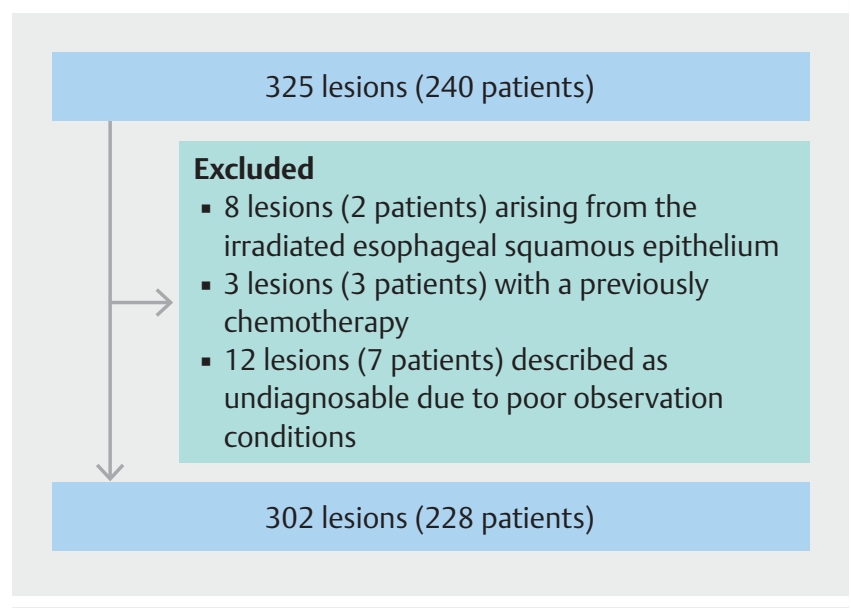

- Fig. 2 Subject selection for this study.

The institutional review board of Fukuoka University of Medicine approved this study protocol (R17-007). The requirement for informed consent for this study was waived because this is a retrospective study dealing with recorded data obtained for the standard clinical practice, that is, preoperative diagnostic endoscopic procedures for superficial esophageal SCC patients.

\section{Analysis group}

Three-hundred twenty-five superficial esophageal SCC lesions from 240 patients were resected in our hospital. We excluded eight lesions from two patients who underwent radiotherapy, three lesions from three patients with previous chemotherapy, and 12 lesions from seven patients whose endoscopic findings could not been fully assessed. Accordingly, 302 lesions from 228 patients were included in the analysis ( $\bullet$ Fig. 2 ). 

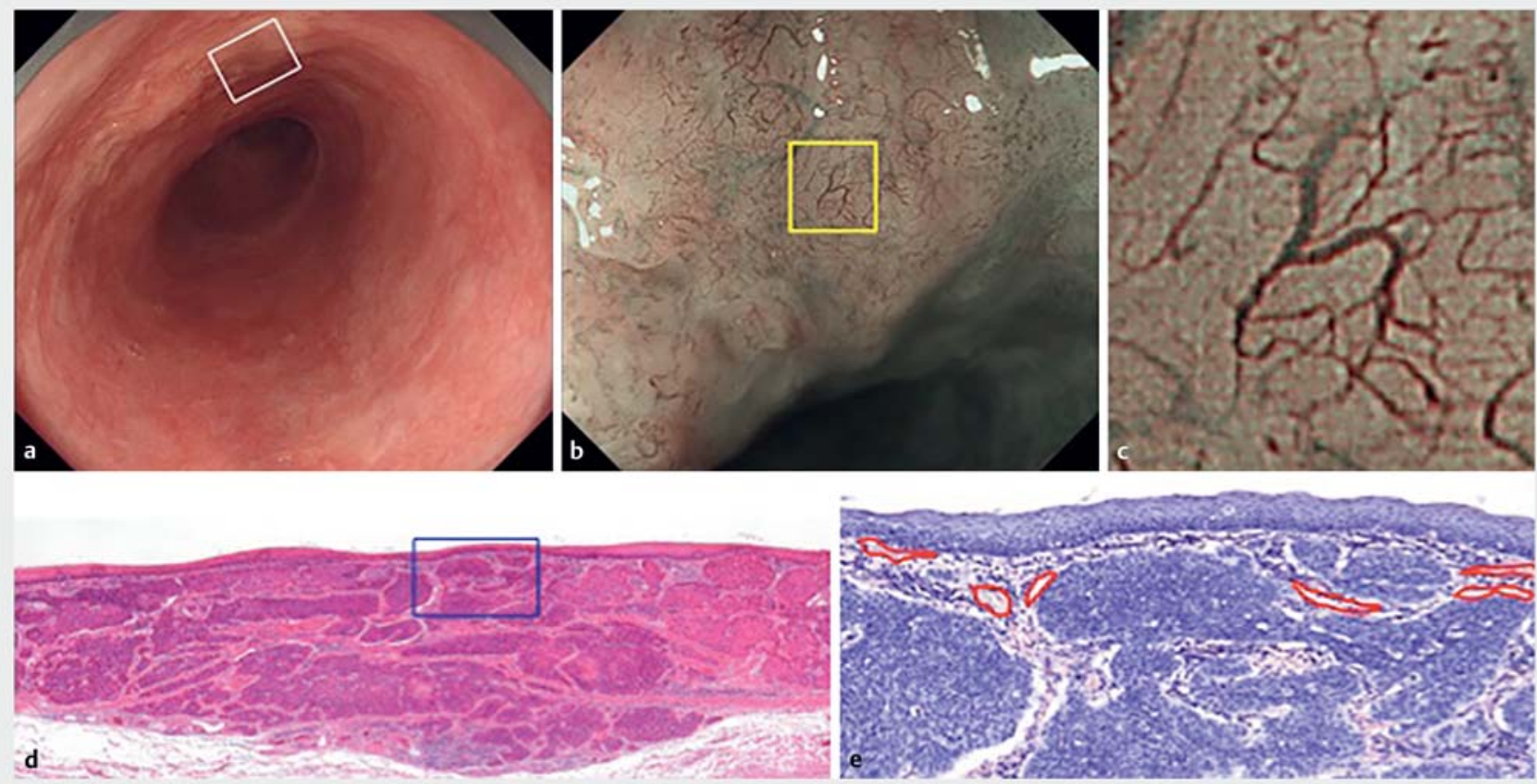

- Fig. 3 Definitions of irregularly branched microvessels. a to $\mathbf{f}$ A case of cancer. Microvessels with an abundance of irregular branches from the dilated abnormal vessels as a trunk. a Endoscopic images obtained using conventional white-light imaging endoscopy show a flat lesion with some slight elevations. b Endoscopic images obtained using M-NBI in the white box of Fig. 3a do not show presence of the B3 vessel. c Endoscopic images obtained using M-NBI in the yellow box of Fig. 3b show irregularly branched microvessels. $\mathbf{d}$ Histopathological findings from the resected specimen. The thin epithelial layer comprised cancerous epithelium. Cancer cells invade the deep submucosal layer. e Histopathological findings from the resected specimen in the blue box of Fig. 3d, showing Factor VIII staining. Vessels with a diameter of 10 to $40 \mu \mathrm{m}$ just beneath the epithelium and between cancer cell nests. We considered that these vessels correspond to irregularly branched microvessels.
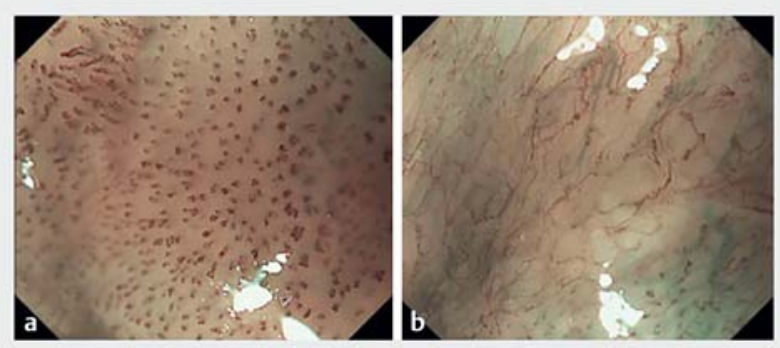

- Fig. 4 a and $\mathbf{b}$ Cases of intramucosal carcinoma. Endoscopic images obtained using M-NBI show the sample without irregularly branched microvessels.

\section{Assessment of microvascular pattern as visualized by M-NBI}

Irregularly branched microvessels were defined as microvessels with an abundance of irregular branches from the dilated abnormal vessels as a trunk visualized by M-NBI. The irregularly branched microvessels differ from the B2 vessel in that they are connected to a trunk. They also differ from the B3 vessel in that they are irregularly branching out from a trunk and are diverse. When we detected at least one irregularly branched microvessel in the cancerous mucosa, irregularly branched micro- vessels were determined as present ( $\bullet$ Fig. $\mathbf{3}$ ). In cases in which any irregularly branched microvessels could not be identified in the cancerous mucosa, irregularly branched microvessels were judged as absent ( $\bullet$ Fig. $\mathbf{4}$ ).

\section{Endoscopic procedure}

All patients underwent optimum preparation by drinking a mixture of mucolytic and deforming agents in water 30 minutes before the procedure. The formula was 20,000 $\mathrm{U}$ of pronase (Kaken Pharmaceutical, Tokyo, Japan), $1 \mathrm{~g}$ of sodium bicarbonate, and $10 \mathrm{~mL}$ of dimethylpolysiloxane $(20 \mathrm{mg} / \mathrm{mL}$; Horii Pharmaceutical, Osaka, Japan) in $100 \mathrm{~mL}$ of water. One milligram of glucagon (Eisai, Tokyo, Japan) or $1 \mathrm{~mL}$ of scopolamine butylbromide $(20 \mathrm{mg} / \mathrm{mL}$; Boehringer Ingelheim, Tokyo, Japan) was routinely administered as an antiperistaltic agent. Most patients underwent sedation by intravenous administration of $5-10 \mathrm{mg}$ of diazepam ( $5 \mathrm{mg} / \mathrm{mL}$; Takeda Pharmaceutical, Tokyo, Japan) or $2-4 \mathrm{mg}$ of midazolam $(10 \mathrm{mg} / 2 \mathrm{~mL}$; Fuji Pharmaceutical Industry, Tokyo, Japan).

\section{Endoscopy system}

All procedures were performed using a high-resolution magnifying upper gastrointestinal endoscope (GIF-Q240Z, GIFH260Z; Olympus Co., Tokyo, Japan) with an electronic endoscopy system (Evis Lucera Spectrum, Olympus Corporation, Tokyo, Japan). 


\section{Handling of resected specimens and histological diagnosis of depth of invasion}

The resected specimens were placed on a flat board with the mucosal side facing upward, pinned at the edges with stainless steel pins, and fixed in a $20 \%$ buffered formalin solution. For endoscopically resected specimens, the fixed specimens were sectioned serially at 2 - to $3-\mathrm{mm}$ intervals. For surgically resected specimens, the fixed specimens were sectioned serially at 2to 5 -mm intervals.

\section{Gold standard}

Histopathological diagnoses of the resected specimen were used as the gold standard.

Histological diagnosis of depth of invasion was performed in accordance with the Japanese classification of esophageal cancer [16]. The pathologist evaluated the invasion depth of superficial esophageal SCC from the resected specimens according to the Japanese classification of esophageal cancer as follows: T1a, tumor invading the mucosa (T1a-EP, carcinoma in situ; T1a-LPM, tumor invading the lamina propria mucosa; T1a-MM, tumor invading the muscularis mucosa); T1b, tumor invading the submucosal layer (SM1, invading to a depth of $\leq 200 \mu \mathrm{m}$ from the muscularis mucosa; T1b-SM2, extending $>200 \mu \mathrm{m})$.

\section{Endoscopic evaluations and endpoints}

Endoscopic findings by $\mathrm{M}-\mathrm{NBI}$ were reviewed by one experienced endoscopist (Y.O.) who was blinded to the histopathological diagnosis and who then determined whether or not irregularly branched microvessels were present. Interobserver agreement (between Y.O. and S.I.) and intraobserver agreement (Y.O.) were assessed. Initially, they examined randomized endoscopic findings by MNBI. Three months later, the same endoscopic findings were reexamined in a different random order.

The primary outcome was diagnostic performance of M-NBI when presence of irregularly branched microvessels was employed as a marker for SM2 cancers. Other outcomes were the additional effect of M-NBI when we used both presence of irregularly branched microvessels as an indicator of SM2 cancers and $\mathrm{B} 3$ vessels, and the interobserver and intraobserver agreements.

\section{Statistical analysis}

Statistical analysis was performed using SPSS software, version 17 (SPSS Inc, Chicago, Illinois, United States). For diagnostic performance, accuracy, sensitivity, specificity, positive predictive value (PPV), and negative predictive value (NPV) are presented as percentages with $95 \%$ confidence intervals (Cls). Continuous variables are expressed as medians and interquartile ranges. Analyses of the difference in diagnostic performance between the B3 vessels and irregularly branched microvessels were conducted using the population whose diagnoses had been confirmed by pathology using the McNemar test. Analyses of the effect of the B3 vessels plus irregularly branched microvessels on diagnostic performance were conducted using the population whose diagnoses had been confirmed by pa- thology and the McNemar test. $P<0.05$ was considered significant.

Interobserver and intraobserver agreements were calculated using the kappa statistic, which determines the agreement beyond that which would be expected due to chance [17]. The kappa statistic was determined for each endoscopist comparing between the first and second observations to estimate the intraobserver agreement and between the two endoscopists for the first trial to estimate the interobserver agreement. Interpretation of the kappa values was performed according to the criteria of Landis and Koch [17], who recommended that a value greater than 0.8 should be considered "almost perfect," between 0.6 and 0.8 "substantial," between 0.4 and 0.6 "moderate," between 0.2 and 0.4 "fair," between 0 and 0.2 "slight," and between 0 and -1 "poor."

\section{Results}

\section{Clinicopathological characteristics of superficial esophageal SCC}

With regard to macroscopic types of the lesions, 65, 44, and 193 lesions were superficial and protruding/slightly elevated type, flat type, and slightly depressed type, respectively. Mean diameter (range) of the lesions was 25.2 (3-110) mm. Regarding the histopathologically determined depth of invasion, 210 , 58 and 34 lesions were determined as EP/LPM, SM1/MM and SM2, respectively. In terms of treatment, 254 lesions were resected endoscopically, 21 lesions underwent ER followed by subsequent chemotherapy or chemoradiotherapy, and 27 lesions were treated by surgery.

\section{Prevalence of irregularly branched microvessels and the $B 3$ vessels according to histopathological depth of invasion}

Prevalence of irregularly branched microvessels in EP-SM1 and SM2 cancers was $4.1 \%(11 / 268)$ and $79.4 \%$ (27/34), respectively, whereas that of B3 was $0.7 \%(2 / 268)$ and $23.5 \%(8 / 34)$, respectively ( $\triangleright$ Table 1 ). In addition, irregularly branched microvessels were present in the lesions where the B3 vessels were detected.

- Table 1 Prevalence of irregularly branched microvessels, the B3 vessels, and histopathological depth of invasion.

\begin{tabular}{|l|l|l|}
\hline & \multicolumn{2}{|c|}{ Histopathological depth of invasion } \\
\hline Microvascular pattern & EP-SM1 $(n=268)$ & $\geq S M 2(n=34)$ \\
\hline $\begin{array}{l}\text { Irregularly branched } \\
\text { microvessels }(n=38)\end{array}$ & $11 / 268(4.1 \%)$ & $27 / 34(79.4 \%)$ \\
\hline \begin{tabular}{l} 
The B3 vessels $(n=10)$ \\
\hline $\begin{array}{l}\text { Irregularly branched } \\
\text { microvessels and/or the }\end{array}$
\end{tabular} & $2 / 268(0.7 \%)$ & $8 / 34(23.5 \%)$ \\
\hline B3 vessels ( $n=38)$ & $11 / 268(4.1 \%)$ & $27 / 34(79.4 \%)$ \\
\hline \begin{tabular}{l} 
EP, epithelium; SM, submucosal layer \\
\hline
\end{tabular}
\end{tabular}


- Table 2 Diagnostic performance of irregularly branched microvessels and the B3 vessels for predicting deep submucosal invasion by carcinoma (SM2).

\begin{tabular}{|c|c|c|c|c|c|}
\hline Microvascular pattern & $\begin{array}{l}\text { Sensitivity } \\
(95 \% \mathrm{CI})\end{array}$ & $\begin{array}{l}\text { Specificity } \\
(95 \% \mathrm{CI})\end{array}$ & $\begin{array}{l}\text { PPV } \\
(95 \% \mathrm{Cl})\end{array}$ & $\begin{array}{l}\text { NPV } \\
(95 \% \mathrm{Cl})\end{array}$ & $\begin{array}{l}\text { Accuracy } \\
(95 \% \mathrm{CI})\end{array}$ \\
\hline Irregularly branched microvessels & $\begin{array}{l}79.4 \\
(66.6-88.4)\end{array}$ & $\begin{array}{l}95.9 \\
(94.3-97.0)\end{array}$ & $\begin{array}{l}71.1 \\
(59.6-79.1)\end{array}$ & $\begin{array}{l}97.3^{1} \\
(95.7-98.5)\end{array}$ & $\begin{array}{l}94.0 \\
(91.1-96.1)\end{array}$ \\
\hline The $B 3$ vessels & $\begin{array}{l}23.5 \\
(14.8-27.7)\end{array}$ & $\begin{array}{l}99.3^{2} \\
(98.1-99.8)\end{array}$ & $\begin{array}{l}80.0 \\
(50.4-94.3)\end{array}$ & $\begin{array}{l}91.1 \\
(90.1-91.6)\end{array}$ & $\begin{array}{l}90.7 \\
(88.8-91.7)\end{array}$ \\
\hline $\begin{array}{l}\text { The B3 vessels and/or irregularly } \\
\text { branched microvessels }\end{array}$ & $\begin{array}{l}79.4^{3} \\
(66.6-88.4)\end{array}$ & $\begin{array}{l}95.9 \\
(94.3-97.0)\end{array}$ & $\begin{array}{l}71.1 \\
(59.6-79.1)\end{array}$ & $\begin{array}{l}97.3^{1} \\
(95.7-98.5)\end{array}$ & $\begin{array}{l}94.0 \\
(91.1-96.1)\end{array}$ \\
\hline \multicolumn{6}{|c|}{$\begin{array}{l}\text { PPV, positive predictive value; NPV, negative predictive value } \\
1 P<0.001 \text { for the B3 vessels vs. the B3 vessels and/or irregularly branched microvessels } \\
2 P<0.05 \text { for the B3 vessels vs. the B3 vessels and/or irregularly branched microvessels } \\
{ }^{3} P<0.001 \text { for the } B 3 \text { vessels vs. the B3 vessels and/or irregularly branched microvessels }\end{array}$} \\
\hline
\end{tabular}

\section{Diagnostic performance of irregularly branched microvessels for SM2 cancers}

When irregularly branched microvessels were used as an indicator of SM2 cancers, the diagnostic accuracy was $94.0 \%(95 \% \mathrm{Cl}$ : 91.4-96.7\%), sensitivity was 79.4\% (95\% Cl: 66.6-88.4\%), specificity was 95.9\% (95\% Cl: 94.3-97.0\%), PPV was 71.1\% (95\% Cl: $59.6-79.0 \%)$, and NPV was $97.3 \%$ (95\% Cl: 05.7-98.5\%) ( $>$ Table 2).

The PPV in two markers did not differ significantly. When we used the irregularly branched microvessels, the NPV significantly improved compared with the use of the B3 vessels alone.

\section{Diagnostic performance of B3 vessels for SM2 cancers}

When B3 vessels were used as an indicator of SM2 cancers, diagnostic accuracy was $90.7 \%$ (95\% Cl: 88.8-91.7\%), sensitivity was $23.5 \%$ (95\% Cl: $14.8-27.7 \%$ ), specificity was $99.3 \%$ (95\% Cl: 98.1-99.8\%), PPV was $80.0 \%$ (95\% Cl: 50.4-94.3\%), and NPV was $91.1 \%$ (95\% Cl: 90.1-91.6\%) (ฉ Table 2 ).

\section{Diagnostic performance of the $B 3$ vessels and/or irregularly branched microvessels for SM2 cancers}

When $\mathrm{B} 3$ vessels and/or irregularly branched microvessels were used as an indicator of SM2 cancers, the diagnostic accuracy was $94.0 \%$ (95\% Cl: 91.4-96.7\%), sensitivity was 79.4\% (95\% Cl: 66.6-88.4\%), specificity was 95.9\% (95\% Cl: 94.3-97.0\%), PPV was $71.1 \%$ (95\% Cl: $59.6-79.0 \%)$, and NPV was $97.3 \%$ (95\% Cl: 05.7-98.5\%) (> Table 2).

B3 vessels and/or irregularly branched microvessels provided significantly better sensitivity for predicting deep submucosal invasion by carcinoma compared with the B3 vessels alone. However, B3 vessels alone provided significantly better specificity for predicting deep submucosal invasion by carcinoma compared with B3 vessels and/or irregularly branched microvessels.

When we used $\mathrm{B} 3$ vessels and/or irregularly branched microvessels as the markers, the NPV was significantly improved compared with the use of $\mathrm{B} 3$ vessels alone.

\section{Interobserver and intraobserver agreements}

The kappa value for interobserver agreement using the marker of irregularly branched microvessels and B3 vessels was 0.531 (moderate agreement) and 0.231 (fair agreement), respectively. The kappa value for intraobserver agreement using the marker of irregularly branched microvessels and B3 vessels was 0.719 (substantial agreement) and 1.00 (perfect agreement), respectively.

\section{Discussion}

This study clearly demonstrated that M-NBI had excellent diagnostic performance when irregularly branched microvessels were used as an indicator of SM2 cancers, with a diagnostic accuracy of $94.0 \%$ (95\% Cl: $91.4-96.7 \%)$, sensitivity of $79.4 \%$ (95\% Cl: 66.6-88.4\%), and specificity of $95.9 \%$ (95\% Cl: $94.3-$ $97.0 \%)$. It was reported that diagnostic accuracy of conventional white-light endoscopy (WLE) and endoscopic ultrasonography (EUS) for diagnosis of SM2 cancer was $61 \%$ to $74 \%$ [18] and 87-90\% [19], respectively. Accordingly, the diagnostic performance of $\mathrm{M}-\mathrm{NBI}$ when we employ irregularly branched microvessels as the diagnostic marker for SM2 cancer was superior or comparable to those of conventional WLE and EUS. In contrast, the marker of the B3 vessels had high specificity (99.3\%) and low sensitivity (23.5\%) as previously reported. Based on this study, sensitivity of presence of $B 3$ vessels used as a marker for SM2 cancer is low. The reason for this is unknown but may be associated with previously reported findings. When tumors reach at least the SM2 stage, not all vessels simultaneously become $60 \mu \mathrm{m}$ in diameter, and the number of such vessels will be small even if that happens. Furthermore, presence of B3 vessels, which are defined as vessels measuring $60 \mu \mathrm{m}$ or more in diameter, is difficult to objectively detect by endoscopy in clinical practice. In contrast, when irregularly branched microvessels were used as a marker, sensitivity of their presence for diagnosing SM2 cancer was relatively high at $79.4 \%$. Although the exact reason for this is unknown, we considered that relative sensitivity increased because irregularly branched microvessels better reflect vascular morphology associated with can- 
cer infiltration, as reported by Arima et al. [11]. Accordingly, it is suggested that when we use irregularly branched microvessels as the marker for SM2 cancers, M-NBI might be useful for accurate differential diagnosis of EP-SM1 cancers from SM2 cancers, thereby contributing to choosing the appropriate therapeutic strategies for the patients.

B3 vessels are defined as highly dilated abnormal vessels with a caliber often larger than $60 \mu \mathrm{m}$ [20]. In this study, the kappa value for interobserver agreement for the B3 vessels was low (0.231), which may be due to the fact that the diameter of the microvessels varies depending on the investigators. In contrast, the kappa value for inter-observer agreement for irregularly branched microvessels was 0.513 , which was better than that of B3 vessels. The decision making according to the morphological characteristic of microvessels, rather than the vessel's diameter, may be more reproducible.

The true mechanism of changes in microvascular architecture is unclear in invasive SCC. Kumagai et al. [21] reported that when the cancerous cells invaded into the part deeper than the mucularis mucosa, the epithelial papilla and capillary are destroyed, and then the newly developed vessels appear dilated and irregularly branched out to the mucosal surface layer. In this study, irregularly branched microvessels were detected closed to the B3 vessels in all lesions where the B3 vessels were detected by M-NBI. We speculate that the neovascular irregular vessels were formed by compression of the invaded cancerous micronests during the cancer invasion process and destruction of squamous epithelial papilla, and then became dilated or tortuous resulting in an irregularly branched morphology as shown in Fig. 3e. Based on histopathological measurements, the diameter of a vessel that was presumed to be the trunk of irregularly branched microvessels was 20 to $40 \mu \mathrm{m}$, and that of the irregularly branched microvessels was 10 to $20 \mu \mathrm{m}$. Because vessels of these sizes cannot be measured by endoscopy, these measurements were not included in the definitions of the irregularly branched microvessels for endoscopy.

In this study, 11 lesions were classified as false positive. For six of them, assessment of EUS or WLE findings in combination with esophageal radiographs did not yield a preoperative diagnosis of SM2 or deeper cancer, and surgical resection was avoided. Thus, in cases of low diagnostic confidence, a comprehensive diagnostic approach combining EUS and other modalities is necessary. On the other hand, the other five lesions were preoperatively diagnosed as SM2 or deeper cancer by other imaging modalities and then surgically resected. They appear to represent the limitations of preoperative diagnosis.

This study had several limitations. First, this research work is a retrospective study based on data obtained in a single center. A prospective study with a multicenter clinical setting is needed to validate the study results. Second, only two expert endoscopists evaluated the M-NBI findings. Non-experts as well as experts from multiple centers should validate diagnostic performance of irregularly branched microvessels. Third, we did not compare diagnostic performance of $\mathrm{M}-\mathrm{NBI}$ with that of EUS.In the near future, we need to perform a clinical study comparing diagnostic performance between $\mathrm{M}-\mathrm{NBI}$ using a marker of irregularly branched microvessel and EUS for predicting SM2 cancers.

\section{Conclusion}

In conclusion, the current study suggests that presence of irregularly branched microvessels visualized by M-NBI may be a useful indicator for accurate diagnosis of superficial esophageal SCC with deep submucosal invasion.

\section{Competing interests}

The authors declare that they have no conflict of interest.

\section{References}

[1] Oyama T, Miyata $\mathrm{Y}$, Shimatani S et al. Lymph nodal metastasis of $\mathrm{m} 3$, sm 1 esophageal cancer (in Japanese with English abstract). Stomach and Intest 2002; 37: 71-74

[2] Tachibana M, Yoshimura H, Kinugasa S et al. Clinicopathological features of superficial squamous cell carcinoma of the esophagus. Am J Surg 1997; 174: 49-53

[3] Endo M, Yoshino K, Takeshita K et al. Analysis of 1125 cases of early esophageal carcinoma in Japan. Dis Esophagus 1991; 4: 125-129

[4] Kodama M, Kakegawa T. Treatment of superficial cancer of the esophagus: a summary of responses to a questionnaire on superficial cancer of the esophagus in Japan. Surgery 1998; 123: 432-439

[5] Araki K, Ohno S, Egashira A et al. Pathologic features of superficial esophageal squamous cell carcinoma with lymph node and distal metastasis. Cancer 2002; 94: 570-575

[6] Eguchi T, Nakanishi Y, Shimoda T et al. Histopathological criteria for additional treatment after endoscopic mucosal resection for esophageal cancer: analysis of 464 surgically resected cases. Mod Pathol 2006; 19: 475-480

[7] Yamashina T, Ishihara R, Nagai K et al. Long-term outcome and metastatic risk after endoscopic resection of superficial esophageal squamous cell carcinoma. Am J Gastroenterol 2013; 108: 544-551

[8] The Japan Esophageal Society. Guidelines for diagnosis and treatment of carcinoma of the esophagus 2017. Tokyo: Kanehara \& Co Ltd; 2017

[9] Takeuchi H, Miyata H, Gotoh M et al. A risk model for esophagectomy using data of 5354 patients included in a Japanese nationwide webbased database. Ann Surg 2014; 260: 259-266

[10] Inoue H. Magnification endoscopy in the esophagus and stomach. Dig Endosc 2001; 13: S40-S41

[11] Arima M, Tada M, Arima H. Evaluation of microvascular patterns of superficial esophageal cancers by magnifying endoscopy. Esophagus 2005; 2: 191-197

[12] Oyama T, Monma K. A new classification of magnified endoscopy for superficial esophageal squamous cell carcinoma. Esophagus 2011; 8: 247-251

[13] Takeuchi M, Hashimoto S, Kobayashi M et al. Prospective study of the usefulness of type B2 vessel determined by the Japan Esophageal Society classification of magnified endoscopy for the diagnosis of superficial esophageal squamous cell carcinoma. Stomach Intest 2014; 49: 164-172 (in Japanese with English abstract)

[14] Fujiwara J, Monma K, Tateishi Y et al. Endoscopic and pathological studies on type B2 blood vessels in estimation of invasion depth of 
superficial esophageal cancer. Stomach Intest 2014; 49: 174-185 (in Japanese with English abstract)

[15] Ikeda H, Inoue H, Sato H et al. Usefulness of a new classification by the Japan Esophageal Society to predict the depth of invasion of esophageal cancer - type B3 vessels. Stomach Intest 2014; 49: 186-195 (in Japanese with English abstract)

[16] Japan Esophageal Society. Japanese classification of esophageal cancer. $11^{\text {th }}$ English edition Tokyo: Kanehara \& Co Ltd; 2015

[17] Landis JR, Koch GG. An application of hierarchical kappa-type statistics in the assessment of majority agreement among multiple observers. Biometrics 1977; 33: 362-374
[18] Chino O, Makuuchi H, Shimada $\mathrm{H}$ et al. Diagnosis of the invasion depth of superficial esophageal carcinoma. Gastroenterol Endosc 2015; 57: 1243-1253 (in Japanese with English abstract)

[19] Murata Y, Napoleon B, Odegaard S. High-frequency endoscopic ultrasonography in the evaluation of superficial esophageal cancer. Endoscopy 2003; 35: 429-436

[20] Oyama T, Inoue H, Arima M et al. Prediction of the invasion depth of superficial squamous cell carcinoma based on microvessel morphology: magnifying endoscopic classification of the Japan Esophageal Society. Esophagus 2016; 13: 138-145

[21] Kumagai Y, Toi M, Kawada K et al. Angiogenesis in superficial esophageal squamous cell carcinoma: magnifying endoscopic observation and molecular analysis. Dig Endosc 2010; 22: 259-267 